\title{
A Hybrid Algorithm for Estimating the Parameters of Polynomial-Phase Signals
}

\author{
Pengfei Tang, Qianqiang Lin, Bin Yuan, Zengping Chen \\ ATR Key Lab, National University of Defense Technology, Changsha, Hunan Prov., China, 410073 \\ E-mail: tangpengfei@nudt.edu.cn
}

\begin{abstract}
This paper details a hybrid algorithm for estimating the parameters of polynomial-phase signals (PPSs) embedded in additive white Gaussian noise. This algorithm combines the high-order ambiguity function (HAF) and the generalized cubic phase function (GCPF), and is a modification of the hybrid CPF-HAF method. In the proposed algorithm, the HAF is first applied on the original PPS to produce a cubic phase signal, whose parameters are then estimated by the GCPF, instead of the CPF. Numerical examples reveals that the signal-to-noise-ratio (SNR) threshold and mean-squared error (MSE) of the proposed approach are significantly lower than the HAF, and the SNR threshold of the proposed approach is increased by about $4 \mathrm{~dB}$ with respect to the CPF$\mathrm{HAF}$, while the MSE is reduced by about $2 \mathrm{~dB}$ above the threshold. In addition, the proposed approach is extended to the product version in the presence of multicomponent PPSs, where the product version of the CPF-HAF suffers from an identifiability problem.
\end{abstract}

Keywords- parameter estimation; polynomial-phase signal; multicomponent signals; high-order ambiguity function; generalized cubic phase function

\section{INTRODUCTIO}

The polynomial-phase signal (PPS) model is widely used for modelling signals in radar, sonar, biomedicine, communications, etc $[1,2,3]$. There are many techniques for estimating the parameters of PPSs. Of these techniques, the best known is the HAF [4]. It is a phase differentiation (PD) technique which decreases the order of the signal phase by one in each stage of the procedure. The resulting signal in the final stage is a complex sinusoid whose frequency is directly proportional to the highest-order phase parameter. With FFT algorithm and one-dimensional search, the highest-order phase parameter can be estimated. The obtained estimate is used to dechirp the original signal and the procedure is repeated until all the parameters are estimated. For an arbitrary Pth-order PPS, the HAF recursively applies PD calculation $P-1$ times. The procedure requires the multiplication of $2^{P-1}$ signal terms, which causes reduced accuracy and numerous cross-terms when multicomponent signals are present [5]. The product highorder ambiguity function (PHAF) [3], which is the extension of HAF, reduces the noise influence and cross-terms by using the product of several HAFs calculated with various lags and proper scaling.

Recently, a simple modification of HAF has been proposed $[6,7]$. When dealing with monocomponent PPS, this approach indeed outperforms the HAF in terms of the accuracy and signal-to-noise-ratio (SNR) threshold.
However, the approach suffers from an identifiability problem when multicomponent PPSs are considered.

The generalized cubic phase function (GCPF) has been proposed for estimating parameters of a cubic phase signal $[8,9]$. Its product version, product GCPF (PGCPF), is capable of dealing with multicomponent cubic phase signals.

In this paper, an approach that combines the GCPF and the HAF has been proposed. In Section II, a short overview of the signal model and the HAF, PHAF, CPF and GCPF techniques is given. In Section III, the proposed GCPF-HAF approach is detailed. Extension to multicomponent PPSs is described in Section IV. In Section V, numerical simulations are carried out to evaluate the performance of the proposed approach. Finally, conclusions are drawn in Section VI.

\section{BRIEF REVIEW OF HAF, PHAF, CPF AND GCPF}

The monocomponent PPS embedded in noise can be modelled as

$$
\begin{aligned}
z_{r}(n) & =z_{s}(n)+z_{w}(n) \\
& =b_{0} \exp \left(j \sum_{i=0}^{P} a_{i} n^{i}\right)+z_{w}(n) \\
- & \frac{N-1}{2} \leq n \leq \frac{N-1}{2}
\end{aligned}
$$

where $z_{w}(n)$ is the additive complex white Gaussian noise with zero-mean and variance $\sigma^{2}, b_{0}$ is the amplitude, $a_{i}, i=0, \ldots, P$, denotes the phase parameters, $N$ is the number of samples and the sampling rate is assumed, without loss of generality, to be unity. The SNR is defined as $S N R=b_{0}^{2} / \sigma^{2}$. The problem of interest is to estimate the parameters $\left\{b_{0} ; a_{i}, i=0, \ldots, P\right\}$ from $z_{r}(n)$.

\section{A. HAF}

For a PPS $z_{s}(n)$, The first-order and second-order instantaneous moments are defined as

$$
\begin{aligned}
& P D^{1}\left(n ; \tau_{1}\right)=z_{s}\left(n+\tau_{1}\right) z_{s}{ }^{*}\left(n-\tau_{1}\right), \\
& P D^{2}\left(n ; \tau_{1}, \tau_{2}\right)=P D^{1}\left(n+\tau_{2} ; \tau_{1}\right)\left[P D^{1}\left(n-\tau_{2} ; \tau_{1}\right)\right]^{*}
\end{aligned}
$$

High-order instantaneous moments (HIM) are defined recursively as

$$
\begin{aligned}
P D^{M}\left(n ; \tau_{1}, \ldots, \tau_{M}\right) & =P D^{M-1}\left(n+\tau_{M} ; \tau_{1}, \ldots, \tau_{M-1}\right) \\
& \times\left[P D^{M-1}\left(n-\tau_{M} ; \tau_{1}, \ldots, \tau_{M-1}\right)\right]^{*}
\end{aligned}
$$

where $M$ is the order of HIM operator and $\tau_{i}$, $i=1,2, \ldots, M$ are the lag parameters. In each stage, the PD 
operator reduces the PPS order by one. Thus $P D^{P-1}\left(n ; \tau_{1}, \ldots, \tau_{P-1}\right)$ of a single component PPS of order $P$ becomes a complex sinusoid with frequency proportional to the highest-order phase parameter

$$
\omega=2^{P-1} P ! a_{P} \prod_{i=1}^{P-1} \tau_{i}
$$

Taking the discrete-time Fourier transform (DTFT) of $P D^{P-1}\left(n ; \tau_{1}, \ldots, \tau_{P-1}\right)$, the $P$ th-order HAF is obtained

$$
H A F(\omega)=\sum_{n=-\left(N / 2-\sum_{i=1}^{P-1} \tau_{i}\right)}^{N / 2-\sum_{i=1}^{P-1} \tau_{i}} P D^{P-1}\left(n ; \tau_{1}, \ldots, \tau_{P-1}\right) \exp (-j \omega n)
$$

By locating the peak of $|H A F(\omega)|$, the highest order phase coefficient $a_{P}$ can be estimated as

$$
\hat{a}_{P}=\frac{1}{2^{P-1} P ! \prod_{i=1}^{P-1} \tau_{i}} \arg \max _{\omega}|\operatorname{HAF}(\omega)|
$$

When $a_{P}$ is obtained, $a_{P-1}$ can be estimated from the dechirped signal $\hat{z}_{s}(n)=z_{s}(n) \exp \left(-j \hat{a}_{P} n^{P}\right)$. The procedure is repeated until all the phase coefficients are estimated.

\section{B. PHAF}

When HAF is applied to multicomponent PPSs, there are introduced a large number of cross-terms that render the parameter estimation ambiguous. The product HAF (PHAF) is proposed to reduce the influence of these cross-terms. It is defined as the product of $L$ HAFs obtained using $L$ different sets of lag parameters, $\tau_{1}^{l}, \tau_{2}^{l}, \ldots \tau_{P-1}^{l}, l=1,2, \ldots, L$, that is

$$
\begin{gathered}
\operatorname{PHAF}(\omega)=\prod_{l=1}^{L} H A F^{l}(\omega), \\
H A F^{l}(\omega)=\sum_{n=-\left(N / 2-\sum_{i=1}^{P-1} \tau_{i}^{l}\right)}^{N / 2-\sum_{i=1}^{P-1} \tau_{i}^{l}} P D^{P-1}\left(n ; \tau_{1}^{l}, \ldots, \tau_{P-1}^{l}\right) \exp \left(-j \omega F_{l} n\right)
\end{gathered}
$$

where $F_{l}=\prod_{i=1}^{P-1} \tau_{i}^{l} / \tau_{i}^{1}$ is the frequency scaling factor. The highest order phase coefficient $a_{P}$ can be estimated as

$$
\hat{a}_{P}=\frac{1}{2^{P-1} P ! \prod_{i=1}^{P-1} \tau_{i}^{1}} \arg \max _{\omega}|\operatorname{PHAF}(\omega)|
$$

\section{C. $C P F$}

The CPF, which was introduced to extract the instantaneous frequency rate (IFR) of a cubic phase signal, is defined as [10]

$$
C P F_{z_{s}}(n, \Omega)=\sum_{m=0}^{(N-1) / 2-|n|} z_{s}(n+m) z_{s}(n-m) \exp \left(-j \Omega m^{2}\right)
$$

It is shown in [11] that for a given value of $n$, the CPF is maximized when $\Omega=2 a_{2}+6 a_{3} n$. Hence, $a_{2}$ and $a_{3}$ can be estimated from the CPF evaluated at two instants, $n=0$ and $n=n_{1}$, as

$$
\begin{aligned}
& \hat{a}_{2}=\hat{\Omega}_{0} / 2 \\
& \hat{a}_{3}=\left(\hat{\Omega}_{n_{1}}-\hat{\Omega}_{0}\right) /\left(6 n_{1}\right)
\end{aligned}
$$

where $\hat{\Omega}_{0}=\arg \max _{\Omega}\left|C P F_{z_{\mathrm{s}}}(0, \Omega)\right|$, and

$$
\hat{\Omega}_{n_{1}}=\arg \max _{\Omega}\left|C P F_{z_{s}}\left(n_{1}, \Omega\right)\right| \text {. }
$$

\section{D. $G C P F$}

GCPF was originally developed in [8] to estimate the parameters of a cubic phase signal. The GCPF is defined as

$$
\begin{aligned}
\operatorname{GCPF}(n, \Omega) & =\sum_{m=0}^{(N-1) / 2-|n|} z_{s}(n+m) z_{s}^{*}(-n+m) \\
& \times z_{s}(n-m) z_{s}^{*}(-n-m) e^{-j 12 n \Omega m^{2}}
\end{aligned}
$$

When the cubic phase signal is substituted into (15), the result is

$$
\begin{aligned}
\operatorname{GCPF}(n, \Omega) & =b_{0} \exp \left[j\left(4 n a_{1}+4 n^{3} a_{3}\right)\right] \\
& \times \sum_{m=0}^{(N-1) / 2-|n|} \exp \left[-j 12\left(\Omega-a_{3}\right) n m^{2}\right]
\end{aligned}
$$

It is obvious that in the absence of noise, $\operatorname{GCPF}(n, \Omega)$ yields a peak at $\Omega=a_{3}$. Hence, $a_{3}$ can be estimated as

$$
\hat{a}_{3}=\arg \max _{\Omega}|\operatorname{GCPF}(n, \Omega)|
$$

When $a_{3}$ is estimated, the other parameters can be estimated by the dechirping technique.

\section{HYBRID GCPF-HAF METHOD}

Since each PD operation in the HAF increases the SNR threshold by approximately $6 \mathrm{~dB}$ and produces additional interference terms, reducing the number of PDs can improve the accuracy and the SNR threshold [12]. The hybrid CPFHAF reduces the number of PDs by two with respect to the HAF, so it has lower SNR threshold and MSE. However, when multicomponent PPSs are considered, there exist multiple peaks for the magnitude of PCPF-HAF at each time instant. Thus, an identifiability problem arises when employing the PCPF-HAF.

For parameter estimation of PPSs of order higher than three, we propose the following two-stage approach which can be referred to as the hybrid GCPF-HAF method. First, we calculate $(P-3)$ th-order PD of the signal $z_{r}(n)$ as [7]

$$
\begin{aligned}
& P D^{P-3}\left[n ; \tau_{1}, \ldots, \tau_{P-3}\right] \\
= & b_{0}^{2^{p-3}} \exp \left\{j \left[C_{3} a_{p} n^{3}+C_{2} a_{p-1} n^{2}\right.\right. \\
+ & \left.\left.\left(C_{1}^{\prime} a_{p-2}+C_{1}^{\prime \prime} a_{p}\right) n+C_{0}^{\prime} a_{p-3}+C_{0}^{\prime \prime} a_{p-1}\right]\right\}+\omega_{z}(n)
\end{aligned}
$$

where

$$
\begin{aligned}
& C_{3}=2^{P-4} \frac{P ! \prod_{i=1}^{P-3} \tau_{i}}{3} \\
& C_{2}=2^{P-4}(P-1) ! \prod_{i=1}^{P-3} \tau_{i}
\end{aligned}
$$

and $\omega_{z}(n)$ is the sum of all noise influenced terms. 
In the second stage, we calculate the GCPF of $P D^{P-3}\left[n ; \tau_{1}, \ldots, \tau_{P-3}\right]$ at time instant $n_{1}$, and estimate the highest-order parameters, $a_{P}$, as

$$
\begin{aligned}
\hat{a}_{P}= & \frac{1}{C_{3}} \arg \max _{\Omega}\left|\operatorname{GCPF}\left(n_{1}, \Omega\right)\right| \\
\operatorname{GCPF}\left(n_{1}, \Omega\right) & =\sum_{m} P D^{P-3}\left(n_{1}+m ; \tau_{1}, \ldots, \tau_{P-3}\right) \\
& \times\left[P D^{P-3}\left(-n_{1}+m ; \tau_{1}, \ldots, \tau_{P-3}\right)\right]^{*} \\
& \times P D^{P-3}\left(n_{1}-m ; \tau_{1}, \ldots, \tau_{P-3}\right) \\
& \times\left[P D^{P-3}\left(-n_{1}-m ; \tau_{1}, \ldots, \tau_{P-3}\right)\right]^{*} e^{-j 12 n_{1} \Omega m^{2}}
\end{aligned}
$$

When $a_{P}$ is estimated, lower order parameters can be estimated by repeating the two stages on the dechirped signal.

The detailed MSE derivation of the GCPF estimator can be derived using the method in [13]. The optimal $n_{1}$ value is given as

$$
n_{1}^{o p t}=\left[0.23\left(N-2 \sum_{i=1}^{P-3} \tau_{i}\right)\right]
$$

where $\lceil\cdot\rceil$ denotes the round up operator.

\section{EXTENSIONS TO MULTICOMPONENT PPSS}

When multicomponent PPSs are considered, the distinct cross-terms occur due to the four-order nonlinearity of the GCPF-HAF. To discern the auto-terms from the cross-terms, the hybrid GCPF-HAF has to be modified.

By using the product and spectral scaling technique similar to the PCPF-HAF, the product version of GCPFHAF can be defined as the product of several hybrid GCPFHAFs calculated with different lag sets

$$
\begin{aligned}
\operatorname{PGCPF}\left(n_{1}, \Omega\right) & =\prod_{l=1}^{L}\left|G C P F_{z_{r}}^{l}\left(n_{1}, \Omega\right)\right| \\
\operatorname{GCPF}_{z_{r}}^{l}\left(n_{l}, \Omega\right) & =\sum_{m} P D^{P-3}\left(n_{1}+m ; \tau_{1}^{l}, \ldots, \tau_{P-3}^{l}\right) \\
& \times\left[P D^{P-3}\left(-n_{1}+m ; \tau_{1}^{l}, \ldots, \tau_{P-3}^{l}\right)\right]^{*} \\
& \times P D^{P-3}\left(n_{1}-m ; \tau_{1}^{l}, \ldots, \tau_{P-3}^{l}\right) \\
& \times\left[P D^{P-3}\left(-n_{1}-m ; \tau_{1}^{l}, \ldots, \tau_{P-3}^{l}\right)\right]^{*} e^{-j 12 n_{1} F_{l} \Omega m^{2}}
\end{aligned}
$$

where $F_{l}=\prod_{i=1}^{P-3} \tau_{i}^{l} / \tau_{i}^{1}$ is the frequency scaling factor and $L$ is the number of different sets of lags. The product and spectral scaling operations ensure the auto-terms are properly aligned and amplified, while the cross-terms are misaligned and weakened. Therefore, the highest-order phase parameter of each component can be estimated by locating the peaks of $\operatorname{PGCPF}\left(n_{1}, \Omega\right)$

\section{NUMERICAL SimULATIONS}

In this section, numerical simulations are provided to illustrate the performance of the proposed method. The number of Monte Carlo simulations is 300 in all examples.
Example 1: In order to compare with other methods, the signal in this example is a six-order PPS with the parameters given by: $b_{0}=1, a_{0}=1.0, a_{1}=\pi / 4, a_{2}=2.1 e-4$, $a_{3}=8.2 e-6, a_{4}=6.2 e-8, a_{5}=2.3 e-10, a_{6}=5.4 e-12$ and the number of samples is 257 . The SNR is varied between 5 and $30 \mathrm{~dB}$. The MSEs of the $a_{6}$ estimate are plotted in Fig.1. From Fig.1, it can be seen that the GCPFHAF has about $5 \mathrm{~dB}$ lower SNR threshold than the HAF and about 4dB higher than the CPF-HAF. Above the threshold, the GCPF-HAF outperforms the HAF and CPF-HAF about $4 \mathrm{~dB}$ and $2 \mathrm{~dB}$, respectively.

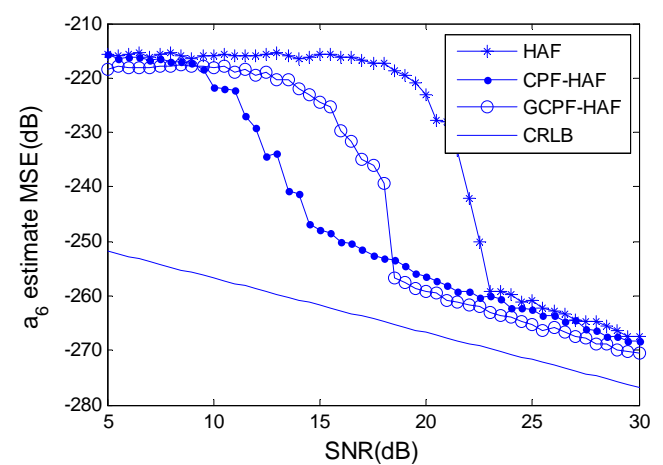

Figure 1. MSEs versus SNR comparison between GCPF-HAF and rival methods for $a_{6}$ estimate of the six-order PPS

Example 2: For multicomponent analysis, the tested signals in this example are the same as the two-component PPS used in [7] except for the amplitudes which are different in [7] to discern the two signals. The first component has parameters given by $b_{0}=1, a_{0}=0, a_{1}=0.77$, $a_{2}=2.41 e-4, a_{3}=-1.61 e-6, a_{4}=-3.34 e-9$, while the second component has parameters given by $b_{0}=1, a_{0}=0$, $a_{1}=-0.83 \quad, \quad a_{2}=-6.82 e-4 \quad, \quad a_{3}=3.94 e-6$, $a_{4}=2.35 e-9$. The number of samples for each component is 1024. The PCPF-HAF is calculated by the product of seven CPF-HAFs, which are calculated using two time instants $n=0, n_{1}^{\text {opt }}=106$ and lags $\tau=\tau^{\text {opt }}+K(l)$, $l=1,2, \ldots, 7$, where $\tau^{o p t}=85$ is set according to [7] and $K(l)=[-38,-26,-4,0,4,26,38]$. When the SNR is $20 \mathrm{~dB}$, the magnitudes of $\operatorname{PCPF}(0, \Omega)$ and $\operatorname{PCPF}\left(n_{1}^{\text {opt }}, \Omega\right)$ are plotted in Fig. 2(a) and Fig. 2(b), respectively. The cubic phase coefficients corresponding to the two components can be estimated by locating two distinct peaks in Fig. 2(a). However, in order to calculate the highest-order coefficient, two peaks should be located in Fig. 2(a) and Fig. 2(b). In this situation, four estimated results are obtained for the highest-order coefficients, and two of the results are spurious. Therefore, the PCPF-HAF suffers from the problem of identifying the parameters when multicomponent PPSs are present.

Fig. 3 shows the magnitude of $\operatorname{PGCPF}(n, \Omega)$ with the lags given by $\tau=\tau^{\text {opt }}+K(l), l=1,2, \ldots, 13$, where 
$K(l)=[-70,-60,-50,-45,-26,-11,0,11,26,45,50,60,70]$ and $\tau^{\text {opt }}=85$. Since $\operatorname{PGCPF}(n, \Omega)$ involves in a four-order nonlinearity, the number of lags used in Fig. 3 is more than that in Fig. 2. The highest-order phase parameters of both PPS components can be estimated by locating two peaks in Fig. 3. Then lower order parameters can be estimated by dechirping and CLEAN techniques [9]. This example reveals that the PGCPF-HAF is capable of dealing with multicomponent PPSs.

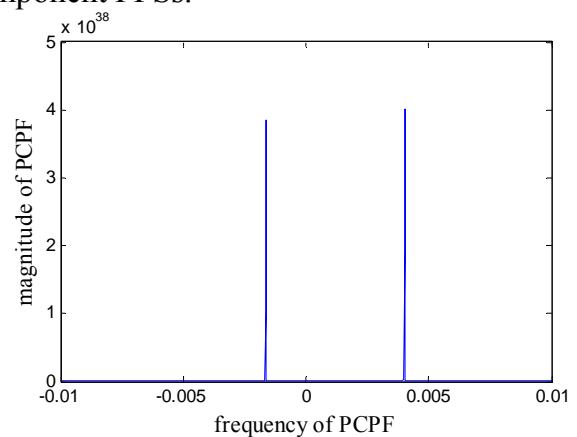

(a)

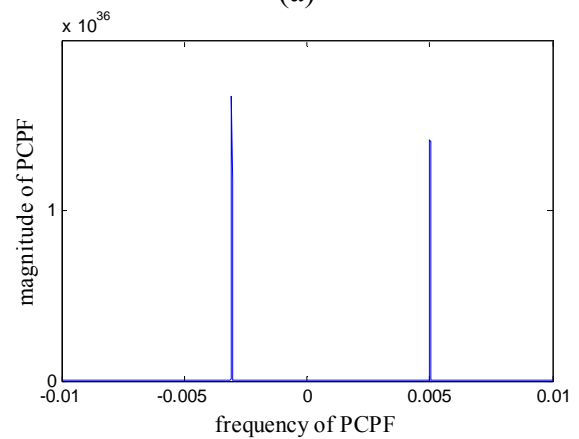

(b)

Figure 2. The magnitudes of PCPF of two-component PPS. (a) The magnitude of $\operatorname{PCPF}(0, \Omega)$, (b) The magnitude of $\operatorname{PCPF}\left(n_{1}^{\text {opt }}, \Omega\right)$.

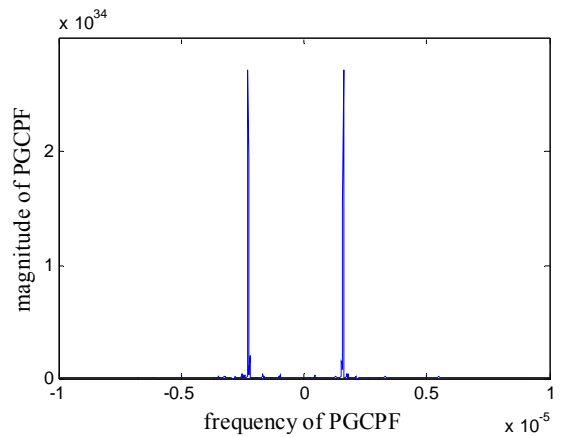

Figure 3. The magnitude of PGCPF of two-component PPS

\section{CONCLUSION}

In this paper, a hybrid algorithm for estimating the parameters of PPSs has been proposed. It is a modification of the hybrid CPF-HAF method and referred to as the hybrid GCPF-HAF method. The proposed algorithm outperforms the HAF in terms of the accuracy and SNR threshold. Since the GCPF involves in a four-order nonlinearity and the CPF employs a bilinear transform, the SNR threshold of the GCPF is slightly higher than the CPF. It should be noted that above the threshold, the MSE of the GCPF-HAF is lower than the CPF-HAF. When multicomponent PPSs are considered, the product version of the proposed algorithm removes the identifiability problem which the product version of the CPF-HAF suffers from. Computer simulations have been carried out to support the theoretical results.

\section{REFERENCES}

[1] Muhammad Z. Ikram, G. Tong Zhou. "Estimation of multicomponent polynomial phase signals of mixed orders", Signal Processing, volume 81, pp. 2293-2308, 2001。

[2] S. Peleg and B. Friedlander, "Multicomponent signal analysis using the polynomial phase transform", IEEE Trans. Aerosp. Electron. Syst., volume 32, pp. 378-387, 1996 。

[3] S. Barbarossa, A. Scaglione, and G. Giannakis. "Product high-order ambiguity function for multicomponent polynomial phase signal modelling", IEEE Trans. Signal Process., volume 46, pp. 691-708, 1998.

[4] S. Peleg, and B. Porat. "Estimation and classification of polynomialphase signals", IEEE Transactions on Information Theory, volume 37, pp. 422-430, 1991

[5] Porat B., Friedlander B. "Asymptotic statistical analysis of the high order ambiguity function for parameter estimation of polynomialphase signals", IEEE Trans. Inform. Theory, volume 42, pp. 9951001, 1996.

[6] M. Simeunović, I. Djurović. "CPF-HAF estimator of polynomialphase signals", Elcetronics Letters, volume 47, 2011.

[7] I. Djurović, M. Simeunović, S. Djukanović, and P. Wang. "A hybrid CPF-HAF estimation of polynomial-phase signals: detailed statistical analysis", IEEE Trans. Signal Process., volume 60, pp. 5010-5023, 2012.

[8] P. O'Shea and R. A. Wiltshire. "A new class of multilinear functions for polynomial phase signal analysis", IEEE Trans. Signal Process., volume 57, pp. 2096-2109, 2009.

[9] Yong Wang and Yicheng Jiang. "Inverse synthetic aperture radar imaging of maneuvering target based on the product generalized cubic phase function", IEEE Geoscience and remote sensing Letters, volume 8, pp. 958-962, 2011.

[10] P. O'Shea. "A new technique for estimating instantaneous frequency rate," IEEE Signal Processing Lett., volume. 9, pp. 251-252, 2002.

[11] P. O'Shea. "A fast algorithm for estimating the parameters of a quadratic FM signal”, IEEE Trans. Signal Process., volume 52, pp. 385-393, 2004.

[12] S. Peleg and B. Porat, "Linear FM signal parameter estimation from discrete-time observations," IEEE Trans. Aerosp. Electron. Syst., volume 27, pp. 607-614, 1991.

[13] P. Wang, I. Djurović, and J. Yang. "Modifications of the cubic phase function", Chinese J. Electron, volume 17, pp. 189-194, 2008. 